\title{
Bi-Directional Reflectance Factor Determination of the Railroad Valley Playa
}

\author{
Carol J. Bruegge ${ }^{1, *(1)}$, Craig Coburn ${ }^{2}$, Arthur Elmes ${ }^{3} \oplus$, Mark C. Helmlinger ${ }^{1}$, Fumie Kataoka ${ }^{4}(0$, \\ Michele Kuester ${ }^{5}$, Akihiko Kuze ${ }^{6}\left(\mathbb{D}\right.$, Tina Ochoa ${ }^{5}$, Crystal Schaaf ${ }^{3}$, Kei Shiomi ${ }^{6}$ and \\ Florian M. Schwandner ${ }^{1,7}$ \\ 1 Jet Propulsion Laboratory, California Institute of Technology, Pasadena, CA 91011, USA; \\ mark.c.helmlinger@jpl.nasa.gov (M.C.H.); temp@gas-monitoring.net (F.M.S.) \\ 2 University of Lethbridge, Lethbridge, AB T1K3M, Canada; craig.coburn@uleth.ca \\ 3 School for the Environment, University of Massachusetts Boston, Boston, MA 02125, USA; \\ Arthur.Elmes@umb.edu (A.E.); Crystal.Schaaf@umb.edu (C.S.) \\ 4 Remote Sensing Technology Center of Japan, Tsukuba-city, Ibaraki 305-0047, Japan; kataoka.fumie@restec.or.jp \\ 5 Maxar (Previously DigitalGlobe), Westminster, CO 80234, USA; Michele.Kuester@maxar.com (M.K.); \\ Tina.Ochoa@digitalglobe.com (T.O.) \\ 6 Japan Aerospace Exploration Agency, Tsukuba 305-8505, Japan; kuze.akihiko@jaxa.jp (A.K.); \\ shiomi.kei@jaxa.jp (K.S.) \\ 7 Earth Science Division, NASA Ames Research Center, Moffett Field, CA 94035, USA \\ * Correspondence: Carol.J.Bruegge@jpl.nasa.gov
}

Received: 27 October 2019; Accepted: 4 November 2019; Published: 6 November 2019

\begin{abstract}
Vicarious calibration is the determination of an on-orbit sensor's radiometric response using measurements over test sites such as Railroad Valley (RRV), Nevada. It has the highest accuracy when a remote sensor's view angle is aligned with that of the surface measurements, namely at a nadir view. For view angles greater than $10^{\circ}$, the dominant error is the uncertainty in the off-nadir correction factor. The factor is largest in the back-scatter principal plane and can reach 20\%. The Orbiting-Carbon Observatory has access to a number of datasets to determine this deviation. These include measurements from field instruments such as the Portable Apparatus for Rapid Acquisition of Bidirectional Observation of the Land and Atmosphere (PARABOLA), as well as satellite measurements from Multi-angle Imaging SpectroRadiometer (MISR) and MODerate resolution Imaging Spectroradiometer (MODIS). The correction factor derived from PARABOLA is consistent in time and space to within $2 \%$ for view angles as large as $30^{\circ}$. Field spectrometer data show that the correction term is spectrally invariant. For this reason, a time-invariant model of RRV surface reflectance, along with empirically derived coefficients, is sufficient to use in the calibration of off-nadir sensors, provided there has been no recent rainfall. With this off-nadir correction, calibrations can be expected to have uncertainties within $5 \%$.
\end{abstract}

Keywords: vicarious calibration; Railroad Valley; RadCalNet; PARABOLA; MISR; MODIS; Orbiting Carbon Observatory (OCO)

\section{Introduction}

Vicarious calibration (VicCal) is one methodology by which the ratio of incident radiance to output digital numbers (DN) can be determined for an on-orbit sensor. This conversion scalar is then used to create future radiance data products. VicCal makes use of surface reflectance and atmospheric transmittance measurements in conjunction with a radiative-transfer code. A preferred test site is Railroad Valley (RRV), Nevada. This dry lakebed is approximately $10 \mathrm{~km}$ in width, homogeneous, and devoid of structures and other developments. The VicCal procedure has been 
used to establish the absolute radiometric scale of a number of sensors, including the Earth Observing System (EOS)/Multi-angle Imaging SpectroRadiometer (MISR) [1]. MISR is a global surveyor that images mid-latitude targets three times per 16-day repeat cycle. It can view RRV at a nadir angle for one of the three paths, and within $10^{\circ}$ for the other two. This is ideal in that the surface reflectance measurements are taken at a nadir-view angle. When the orbital sensor views at the same angle as the surface measurements, there is no need to correct the field observations for an off-nadir view angle. Conversely, calibration of Orbiting Carbon Observatory-2 (OCO-2) is more challenging [2], due to both the need to schedule observations, and the large view angles that often accompany these observations. View zenith angles range up to $20^{\circ}$, for which the surface reflectance changes by as much as $6 \%$ compared to the nadir-view value. Likewise, Japan's Greenhouse gasses Observing SATellite (GOSAT) views at large off-nadir angles. Specifically, the view from Path 36 is from the East at $20^{\circ}$ zenith $(20 \mathrm{E})$, the forward-scattering direction, and from the West at $30^{\circ}$ zenith $(30 \mathrm{~W})$ for Path 37 , the backscatter direction. The overpass times for both OCO-2 and GOSAT is early afternoon, at approximately 21:00 (Coordinated Universal Time (UTC) (14:00 Pacific Daylight Time (PDT)).

The off-nadir deviation in reflectance can be determined by a number of ground and orbital instruments. Ground instruments include the Portable Apparatus for Rapid Acquisition of Bidirectional Observation of the Land and Atmosphere (PARABOLA), the University of Lethbridge Goniometer System-2 (ULGS-2), and Analytical Spectral Devices (ASD) FieldSpecPro field spectrometers (Longmont, CO, USA). Orbital sensors with relevant datasets are from Multi-angle SpectroRadiometer (MISR) and MODerate resolution Imaging Spectroradiometer (MODIS). Historically, the OCO and GOSAT communities have relied upon the MODIS surface product. This is because of its global coverage, and the fact that the product is updated daily using data from a morning and an afternoon sensor. As we will show, this standard product fails to retrieve surface reflectances over our desert test sites. This is due to a data quality flag confusing the bright service with a hazy atmosphere. The research reported here validates better alternatives. Both MISR data, with new data available three times in 16 days, or the PARABOLA model reported here are appropriate datasets.

RRV is a valued VicCal test site, due to its size and lack of development. It is under Bureau of Land Management (BLM) stewardship, a Federal agency whose mission is to coordinate shared usage of public land. Both National Aeronautics and Space Administration/Jet Propulsion Laboratory (NASA/JPL) and the University of Arizona, Tucson, have land rights-of-way with the BLM, in order to place network sensors on the playa. The surface of the RRV playa is spatially and spectrally homogenous, with slight variations on the scale of several hundreds of meters extent. The surface optical characteristics are largely determined by three factors: surface mineral composition, surface fine structure, and soil moisture. Mineralogically, the surface composition is dominated by a mixture of clay minerals (tan colors), fine grained quartz crystals (white blotches), rock microlites (black spots, mostly an artifact of from surface road engineering), and coarse sand-sized quartz. Salts are less common but have been found to form clusters at 10-20 cm depth. The compositional endmembers of these components vary across the playa, as does the surface structure, including mud cracks and greater angular variability in softer substrate locations. Frequent winds during summer afternoon campaigns tend to concentrate in the eastern part of the playa, and, in some years, higher surface concentrations of sand-sized quartz grains have been found at some locations, like M03, likely affecting the overall optical characteristics of the surface. After rain events, surface albedo has been found to stabilize after approximately 1-2 weeks of dry conditions. Being a desert playa in a locally arid environment, sabkha processes dominate soil moisture [3] including evaporative pumping, which causes a dryer surface during the day at the same time experiencing a higher evaporative throughput of water from below. The combination of these compositional, hydrological, and structural effects could cause differences in the apparent bi-directional reflectance factor (BRF), but the off-nadir reflectance, normalized to its value at nadir, is shown here to have minimal variability in time and location within the playa. 
Off-nadir BRF is also of interest to those making sensor cross-comparisons, where two sensors are likely be looking at an unvegetated Pseudo-Invariant Calibration Sites (PICS) target at different view and/or sun angles. In this application, a correction is needed to adjust the radiances measured by the reference sensor into effective radiances that would have been seen at the view angles of the sensor to be calibrated. This technology is not specific to RRV, but the validation of the MISR BRF data product over desert sites, as shown here, is relevant to those making these cross-sensor comparisons.

\section{Vicarious Calibration}

The calibration of a nadir-viewing on-orbit sensor is performed by having it record data over a test site, such as RRV, simultaneous to making in situ measurements. At RRV, an Aerosol Robotic Network (AERONET) Cimel instrument records aerosol optical depth (AOD) and its slope with wavelength. If the experiment is done with use of a staffed field-campaign, then surface reflectance measurements are made with a spectrometer, typically an ASD FieldSpecPro. These data are collected with a nadir-viewing optic, as it would difficult to collect data at all possible sensor view angles. Hundreds of data samples are collected, all within a half hour of the satellite overpass. The time window is necessary in that the reflectance will change with a change in the incident sun angle. Periodically, the spectrometer is used to collect data over a reflectance standard, including a measurement at the start and end of the window. Preferred is a Spectralon reflectance standard, which approaches $100 \%$ reflectance in the visible and near-infrared, and is near Lambertian (diffuse). The spectrometer need not be calibrated for this application, as traceability to an International System of Units (SI) is via the reflectance standard. The reflectance is computed by correcting the Spectralon data for its spectral reflectance and $\mathrm{BRF}$, interpolating to a data value at the time sample of the target (playa), then taking the ratio of target to adjusted Spectralon. These are averaged to obtain a reflectance representative of an area within the flight-sensors field-of-view. All inputs (including AOD, reflectance) are input into a radiative transfer code, such as MODTRAN, and used to derive the top-of-atmosphere (TOA) radiance incident at the sensor. Radiometric calibration coefficients are determined from this TOA radiance and the sensor's digital number output. The calibration equation used is specific to the sensor under test.

In the absence of a field team, network data can be used to determine surface reflectance. Data derived from the in situ instruments at RRV are accessible to the public via the on-line RadCalNet portal [4,5]. TOA radiances are available at a nadir-view angle, as well as the bottom-of-atmosphere reflectances. These are reported at $10 \mathrm{~nm}$ sampling, from 400 to $1000 \mathrm{~nm}$, and from 9:00 UTC. to 15:00 UTC. local time. With these, calibration scientists have access to datasets that can be used to calibrate nadir-viewing sensors. For sensors that view off-nadir, RadCalNet data alone are not sufficient.

For vicarious calibration of off-nadir sensors, the absolute BRF (reflectance with angle) is not required, but rather a relative factor normalized to the nadir view. Mathematically, we write:

$$
\begin{gathered}
\operatorname{BRF}(\text { Ssun; Sview })=\operatorname{BRFasd}\left(\text { Ssun; } 0^{\circ}\right) * \text { normBRF, where } \\
\text { Ssun }=\theta \text { sun, } \phi \text { sun, Sview }=\theta \text { view, } \phi \text { view, and } \\
\text { normBRF }=\operatorname{BRF}(\text { Ssun; Sview }) / \operatorname{BRF}\left(\text { Ssun; } 0^{\circ}\right) .
\end{gathered}
$$

In Equation (1), we see that the surface reflectance used in the vicarious calibration analysis contains the parameter, BRFasd, which is measured by the field team using an ASD. These data are taken at a nadir view, hence the term BRFasd (Ssun; $0^{\circ}$ ), where the function varies with $S_{\text {sun, }}$ the solar zenith, and azimuth angle (Equation (2)). This is multiplied by normBRF, derived from PARABOLA or other source. In Equation (3), we define the normalized BRF, normBRF, as the off-nadir BRF, at the solar-incident and view angles of interest, divided by the nadir BRF. This parameter has a value of 1 for a nadir viewing sensor, and otherwise describes the deviation with view angle, compared to a nadir view. For sensors that view close to nadir, the normBRF correction term is small. However, it can be large for the GOSAT and OCO sensors. Once the normBRF is determined, it multiplies the ASD 
measured (or RadCalNet) surface reflectance. A radiative transfer code is then used to compute the TOA radiance incident on the flight sensor, and the calibration is completed.

VicCal measurements of top-of-atmosphere radiance are typically quoted as having an uncertainty of $3 \%(1 \sigma)$ for nadir-viewing sensors [6]. We require that VicCal for the OCO and GOSAT sensors to have an uncertainty no larger than $5 \%$, for views as large as $30^{\circ}$ off-nadir $[7,8]$. This drives the need to determine the uncertainties in the off-nadir datasets.

\section{PARABOLA}

The PARABOLA [9], Figure 1, is a fully automated sphere-scanning radiometer consisting of eight spectral channels. Four of these $(444,551,650$, and $860 \mathrm{~nm})$ provide data with MISR passbands. Additionally, one channel exists for the characterization of water vapor (944 nm), and two for characterization of aerosol optical depth at longer wavelengths (1028 and $1650 \mathrm{~nm})$. The latter channel is also used for vegetation studies. Finally, a broad-band channel (400-700 nm, centered at $581 \mathrm{~nm}$ ) provides measurement of photosynthetic active solar radiation (PAR). PARABOLA's azimuth and zenith drives allow sampling at $5^{\circ}$ steps in both the upper and lower hemispheres, with $5^{\circ}$ fields-of-view. This generates 2664 samples ( 37 zenith by 72 azimuth) during the scan repeat time of 3.3 minutes. The silicon and germanium detectors, the latter for the 1028 and $1650 \mathrm{~nm}$ channels, are all thermoelectrically cooled to $-10^{\circ} \mathrm{C}$. As we are interested in surface reflectance, only data from surface-leaving, upwelling light, are discussed here. The downwelling light is used in the analysis to orient the data product to North for a $0^{\circ}$ azimuth angle.

In total, 34 PARABOLA datasets have been collected at RRV between 2011 and 2019. Beginning in 2013, the locations have been set at four sites: M03 (38.4839 N, 115.6849 W), M20 (38.4530 N, 115.6440 W), MDN $(38.4991 \mathrm{~N}, 115.6917 \mathrm{~W})$, and H14 $(38.4396 \mathrm{~N}, 115.6687 \mathrm{~W})$, all at a surface elevation of $1437 \mathrm{~m}$. The distance from MDN, to the North, and H14, the furthest to the south, is $7.1 \mathrm{~km}(4.4 \mathrm{mi})$. At each of these sites, a permanently installed telescoping mast allows easy installation and repeatability of location. The instrument is telescoped up to a height of roughly $4.6 \mathrm{~m}(15 \mathrm{ft})$. Surface images from two of these sites are shown in Figure 2. The northern most sites, MDN and M03, are soft. Footprints at these locations sink to several centimeters or so in depth. The southern two sites are hard and flat. No footprints are left at these locations.
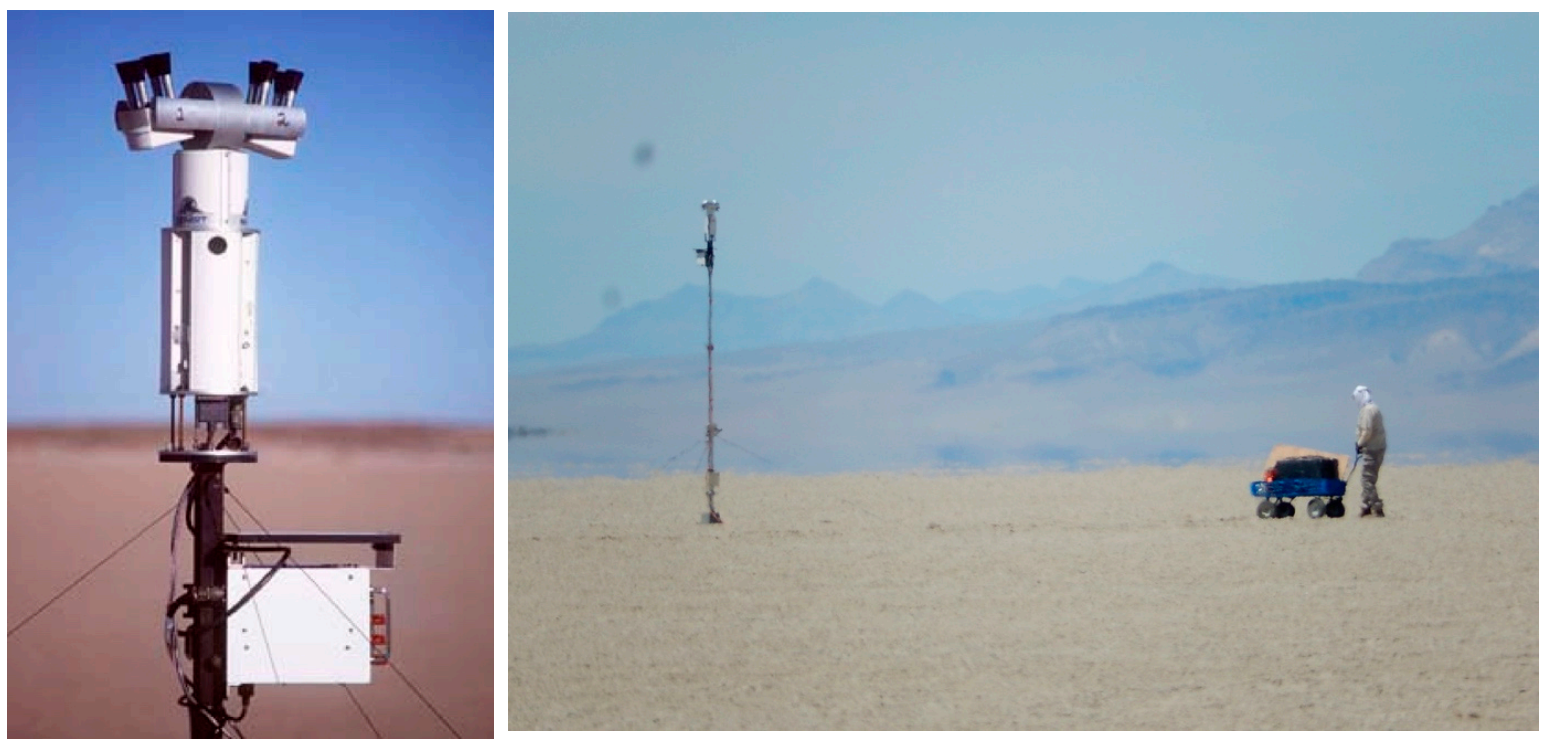

Figure 1. The Portable Apparatus for Rapid Acquisition of Bidirectional Observation of the Land and Atmosphere (PARABOLA) 8-channel hemispheric scanning radiometer. (Left) instrument closeup, where a Spectralon diffuse reflectance standard sits on top of the electronics module and is viewed during the nadir scan; (Right) instrument at a final height, with a field engineer for perspective. 

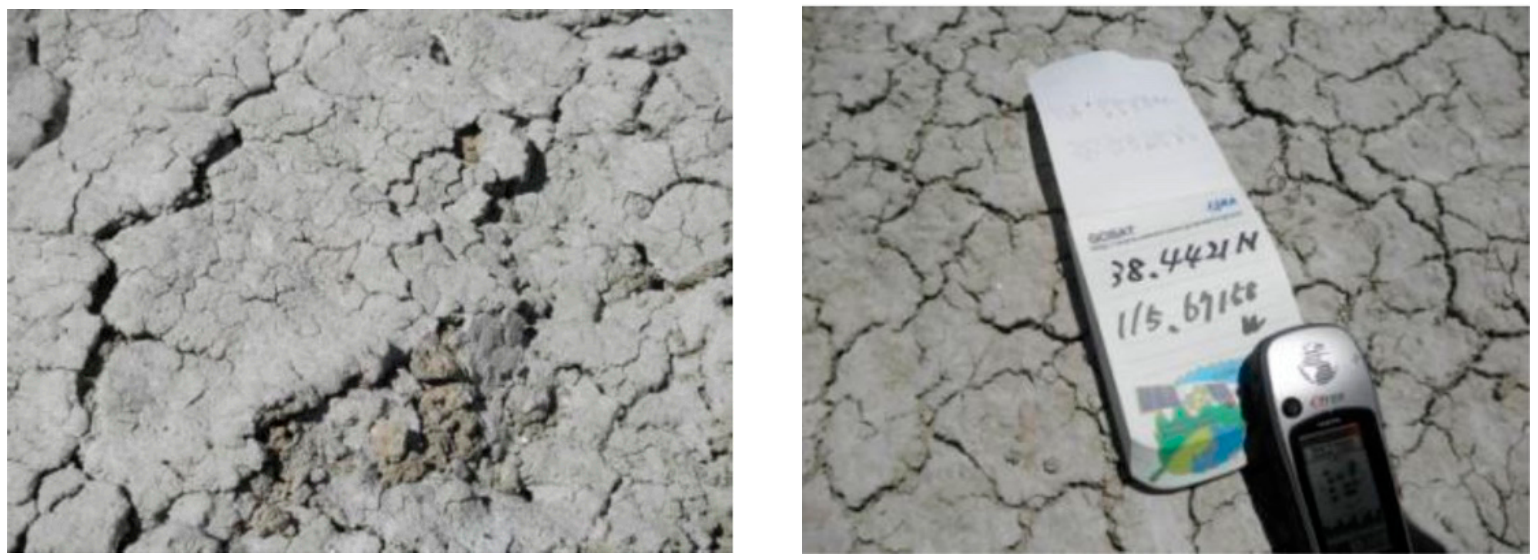

Figure 2. Surface photos taken at: (Left) M03, a soft surface, and (Right) H14, a hard surface.

The instrument is set up in the morning, and taken down late afternoon, unless high winds or clouds call for an early termination for the day. Figure 3 (left) shows the raw data for the $581 \mathrm{~nm}$ channel. The center of this polar plot represents the nadir view. Moving out in radius from here are views from nadir to the horizon $\left(\right.$ at $\left.90^{\circ}\right)$, with dotted lines at $30^{\circ}$ and $60^{\circ}$ view zeniths. The solar azimuth for this scan (taken at 21:05 UTC) was at $23^{\circ}$ zenith and $233^{\circ}$ azimuth. The blue/green line appearing opposite the solar azimuth $\left(\right.$ at $\left.53^{\circ}\right)$ and originating from the center is the mast shadow. It is observed that there is a "hotspot" retroreflection, shown in red, in the backscatter direction. This hotspot is due to the small-scale topography, and the fact that the illumination of surfaces facing the sun are brighter than those that are inclined at a different angle. The bright (red) pixels from azimuth $180^{\circ}$ to $225^{\circ}$ and extending to about $20^{\circ}$ zenith are due to the presence of a Spectralon reflectance standard.
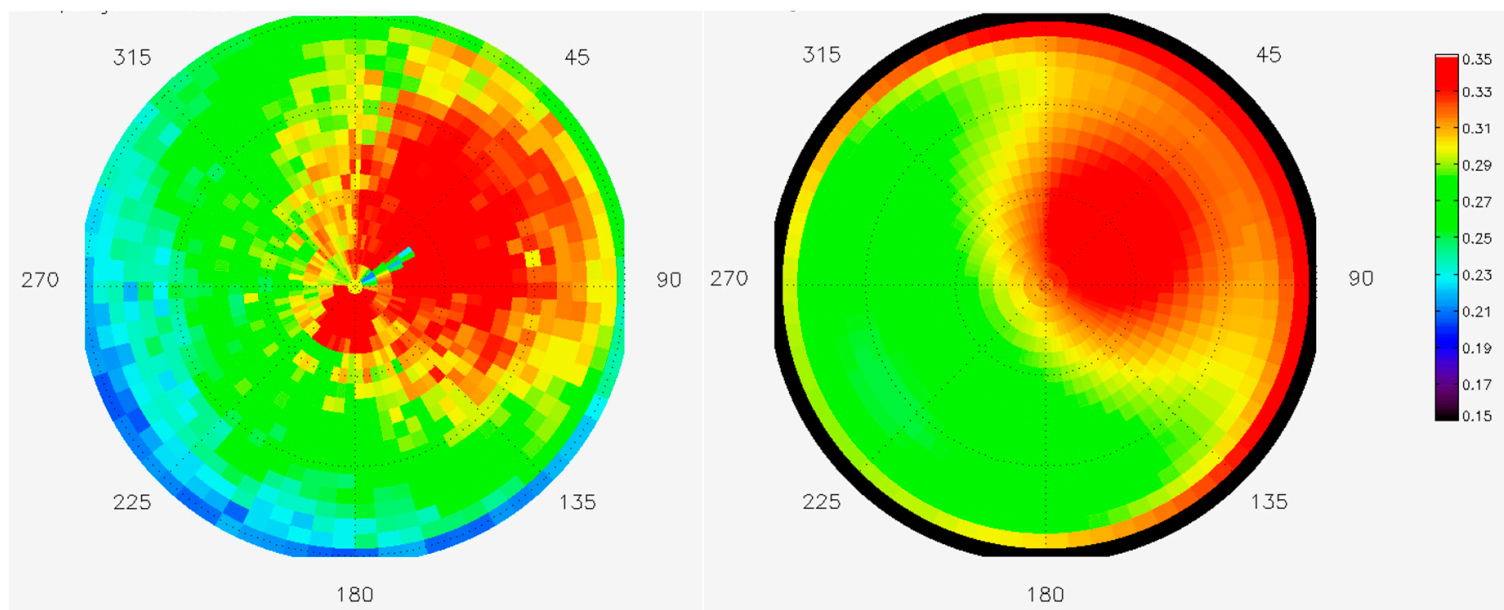

Figure 3. (Left) PARABOLA raw data as acquired on 28 June 2018, $581 \mathrm{~nm}$ band. The Sun is in the southwest, and the hotspot is in the northeast in the back-scatter direction; (Right) data smoothed following a modified Rahman-Pinty-Verstraete (mRPV fit).

In this paper, we refer interchangeably to either the surface bi-directional reflectance factor (BRF) or bi-directional reflectance distribution function (BRDF). These functions are reported by projects as a means to describe land surface reflectance anisotropy. They are used to determine leaf-area index, surface roughness, snow grain size, land surface albedo, and more. The first is the ratio of upwelling radiance to that from to an ideal (100\% reflecting, perfectly diffuse) standard, under the same illumination and viewing conditions. The latter is the ratio of reflected radiance to downwelling irradiance. As the Lambertian reference target is ideal, the light reflected from it is scattered equally into $\pi$ steradians, hence it can be shown that $\mathrm{BRF}=\pi^{*} \mathrm{BRDF}$. Because BRF is a ratio of two fluxes, it is a 
unitless quantity, unlike BRDF. Aside from the factor of $\pi$, the BRF and the BRDF are essentially identical descriptions of the scattering properties of a surface, and the two terms can be used interchangeably. A discussion of this and reflectance terminology in general can be found in [10]. Those familiar with these terms might also clarify that it is the hemispheric-directional reflectance factor (HDRF) that is measured here. This is because light strikes the surface from all angles, an effect due to atmospheric scattering. The difference between BRF and HDRF has been shown to be small for clear sky desert conditions, as is found at RRV. Thus, here we approximate BRF from the measured HDRF, and do not refer to HDRF further.

The first processing step is to rotate the data such that a view azimuth of $0^{\circ}$ refers to a sensor looking from the North. This is done by comparing the location of the sun, in the upwelling observations, to that expected from a solar ephemeris look-up. An offset clocking value is determined in this step, and applied as a geometric calibration scalar. Next, the data at all view angles are ratioed to the data at nadir, which is the Spectralon view. This produces a BRF, except at view angle locations that are not viewing the target of interest. For these samples, such as those containing the Spectralon and mast shadow, the BRF is approximated with values extrapolated from a polynomial fit along zenith slices. The data are then smoothed by fitting to the modified Rahman-Pinty-Verstraete (mRPV) surface model [11], with results shown in Figure 3 (right). The model has three free parameters, $r_{0}, k$, and b, and is given in Equations (4)-(7). Here, $\theta_{0}$ is the solar zenith angle, $\theta$ the view zenith, and $\phi$ the view azimuth relative to the sun. The function $h$ is a factor to account for the hot spot. The function $\cos \xi$ depends only on scattering angle, the angle between the directions of the incident, and reflected radiances. This model is physically based, and the description of the hot spot is derived from the transmission of radiation in a porous medium, and it has been widely used and validated for a variety of soil and vegetation surfaces [12]:

$$
\begin{gathered}
B R F\left(\theta_{0}, \theta, \phi\right)=r_{0}\left(\cos \theta \cos \theta_{0}\left(\cos \theta+\cos \theta_{0}\right)\right)^{k-1} h \exp (-b \cos \xi), \\
h\left(\theta_{0}, \theta, \phi\right)=1+\frac{1-r_{0}}{1+G\left(\theta_{0}, \theta, \phi\right)} \\
G\left(\theta_{0}, \theta, \phi\right)=\sqrt{\tan ^{2} \theta+\tan ^{2} \theta_{0}-2 \tan ^{2} \theta \tan ^{2} \theta_{0} \cos \phi} \\
\cos \xi=\cos \theta \cos \theta_{0}+\sin \theta \sin \theta_{0} \cos \phi .
\end{gathered}
$$

We wish to explore this normalized function, normBRF, as a function of view angle, wavelength, location, and time. We focus on the 21:00 UTC (14:00 PDT) overpass time, with a solar zenith-23 in summer, and the $30 \mathrm{~W}, 20 \mathrm{E}$ view angles, as these of interest to the OCO and GOSAT communities. For the above plot, the normBRF value are respectively 1.05 and 0.91 for the backscatter and forescatter directions, respectively, in the West-East azimuthal scan. These numbers are invariant over the PARABOLA bands, except for the water band where there is a $1.9 \%$ deviation from these values.

We next look at the deviation in normBRF over time for each of our four RRV sites. Table 1 gives the $r_{0}, k, b$ coefficients for a typical collect at each of the four test sites-the campaign date was chosen as that representing an average profile over several different field campaign dates. Here, the normBRF values at the GOSAT angles of $30 \mathrm{~W}$ and $20 \mathrm{E}$ are reported. The agreement at $30 \mathrm{~W}$ is within 0.01 and at $20 \mathrm{E}$ within 0.02 . This suggests that a fixed parameter set, such as that from 20180628/MDN, might represent the playa within the area covered by our four test sites. The largest percentage deviation from the average is reported in parentheses for a given site. For example, at MDN, there were nine full data datasets acquired between 2013 and 2019. Using the data from each day where the solar zenith angle is closest that of 28 June 2018 ( $23^{\circ}$ zenith), the $\mathrm{r}_{0}, \mathrm{k}$, and b coefficients were computed. These were then used to calculate normBRF at $30 \mathrm{~W}$ and $20 \mathrm{E}$. The largest deviation, from the value at 28 June 2018, among these nine data sets was $2 \%$ at $30 \mathrm{~W}$ and $1.3 \%$ at $20 \mathrm{E}$. Assuming these deviations are due to surface changes, using the model derived from 28 June 2019 would be acceptable, allowing us to meet our VicCal uncertainty requirement. 
Also included in Table 1 is a retrieval over Frenchman Flat $\left(36.8093^{\circ} \mathrm{N}, 115.9348^{\circ} \mathrm{W}, 940 \mathrm{~m}\right)$, a hard, small playa on the Nevada Test Site (NTS). This surface is seen to be brighter (as shown by the $\mathrm{r}_{0}$ coefficient) and more Lambertian. It has a normBRF that is $4 \%$ lower than Railroad Valley at $30 \mathrm{~W}$ and $7 \%$ higher at $20 \mathrm{E}$, in both cases implying it is closer to Lambertian. As expected, these harder, flatter surfaces have less variation with angle at these high sun angles. At low sun angles, we see a specular term not seen at RRV.

Table 1. Normalized bi-directional reflectance factor (normBRF) at $581 \mathrm{~nm}$ at two view zenith angles in the $90^{\circ}$ from North azimuth. In parentheses are the largest deviation from the reported normBRF, over the annual collects at the specified site, for a comparable sun angle.

\begin{tabular}{cccc}
\hline Date/Site & $\mathbf{r}_{\mathbf{0}}, \mathbf{k}, \mathbf{b}$ & $\mathbf{3 0}^{\circ} \mathbf{W}$ (Deviation) & $\mathbf{2 0}^{\circ} \mathbf{E}$ (Deviation) \\
\hline 20180628/MDN & $0.179,0.800,-0.254$ & $1.080(2.1 \%)$ & $0.910(1.3 \%)$ \\
$20131020 / \mathrm{M} 03$ & $0.210,0.835,-0.235$ & $1.070(1.2 \%)$ & $0.912(1.8 \%)$ \\
$20150628 / \mathrm{M} 20$ & $0.206,0.783,-0.148$ & $1.079(0.5 \%)$ & $0.929(1.5 \%)$ \\
$20180701 / \mathrm{H} 14$ & $0.183,0.800,-0.291$ & $1.081(0.8 \%)$ & $0.905(3.1 \%)$ \\
$20130415 / \mathrm{NTS}$ & $0.368,0.889,0.120$ & 1.041 & 0.971 \\
\hline
\end{tabular}

In looking at normBRF retrievals, from the PARABOLA, we note that data from the $444 \mathrm{~nm}$ channel are suspect, due to a low SNR. Additionally, we see that the $650 \mathrm{~nm}$ channel is no longer operational, beginning in 2018.

\section{University of Lethbridge Goniometer System (ULGS)}

From 1-5 May 2018, a group of scientists gathered at RRV, in order to intercompare methods and measurements. In addition to the PARABOLA, the ULGS-2 was present for the campaign. The ULGS system consists of two Ocean Optics USB4000 spectrometers: one mounted to a goniometric arm and the second measuring downwelling light. The standard data collection mode for the ULGS-2 involves averaging the signal over the $400-1000 \mathrm{~nm}$ range into $10 \mathrm{~nm}$ spectral bands for analysis. An ASD FieldSpecPro additionally measures nadir reflectances from 350-2500 nm, using an $8^{\circ}$ FOV at $1 \mathrm{~m}$ height above ground.

To process the data, the first bad samples are removed. For example, the goniometer arc creates a shadow in the solar principal plane and must be removed. The goniometer data are scaled such that they agree with the ASD data at nadir. A figure of the instrument, and a polar plot of the data are shown in Figure 4.
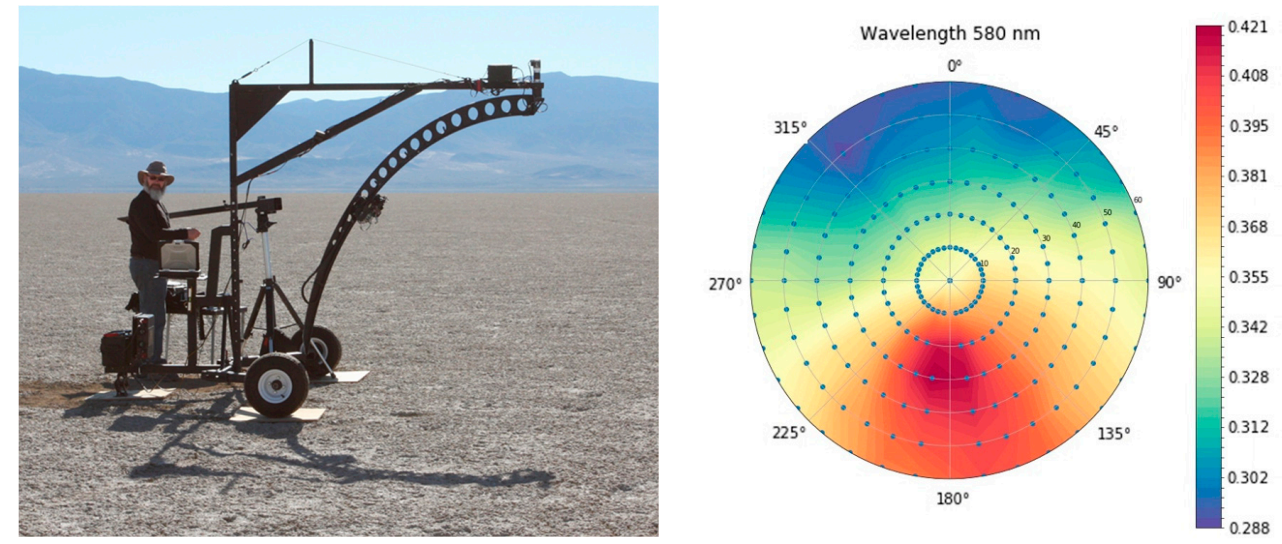

Figure 4. (Left) Univ. of Lethbridge Goniometer System. (Right) BRF at 580 nm, 03 May 2018, 20:00 UTC. $0^{\circ}-180^{\circ}$ is the principal plane with the forward scatter (less reflective) at the top.

The so-called "Golden Day" for this campaign was 3 May 2018. On this day, the skies cleared, in an otherwise cloudy campaign week. The aerosol optical depth was 0.07 at $500 \mathrm{~nm}, 20: 00 \mathrm{UTC}$, as recorded by the Aerosol Robotic Network (AERONET) Cimel. This is slightly higher than the clearest summer 
days, were values are as low as 0.04 , but not hazy enough to compromise the retrieval. Nineteen goniometric scans were made on this day, from 14:30-22:27 UTC (7:30-15:00 PDT). A comparison of the ULGS results with PARABOLA is shown in Figure 5. The two instruments were separated by $0.9 \mathrm{~km}$ $(0.5 \mathrm{mi})$. The figure shows that the ULGS and PARABOLA normBRF values are roughly comparable. For a relative azimuth of $80^{\circ}$, simulating GOSAT overpass conditions, the fractional difference between the two normBRF reports are 0.06 at $30 \mathrm{~W}$ and 0.005 at $20 \mathrm{E}$, at $580 \mathrm{~nm}$. At wavelengths above $900 \mathrm{~nm}$, the Ocean Optics USB4000 spectrometer suffers from a low signal-to-noise ratio (SNR), and thus the data are not reported.
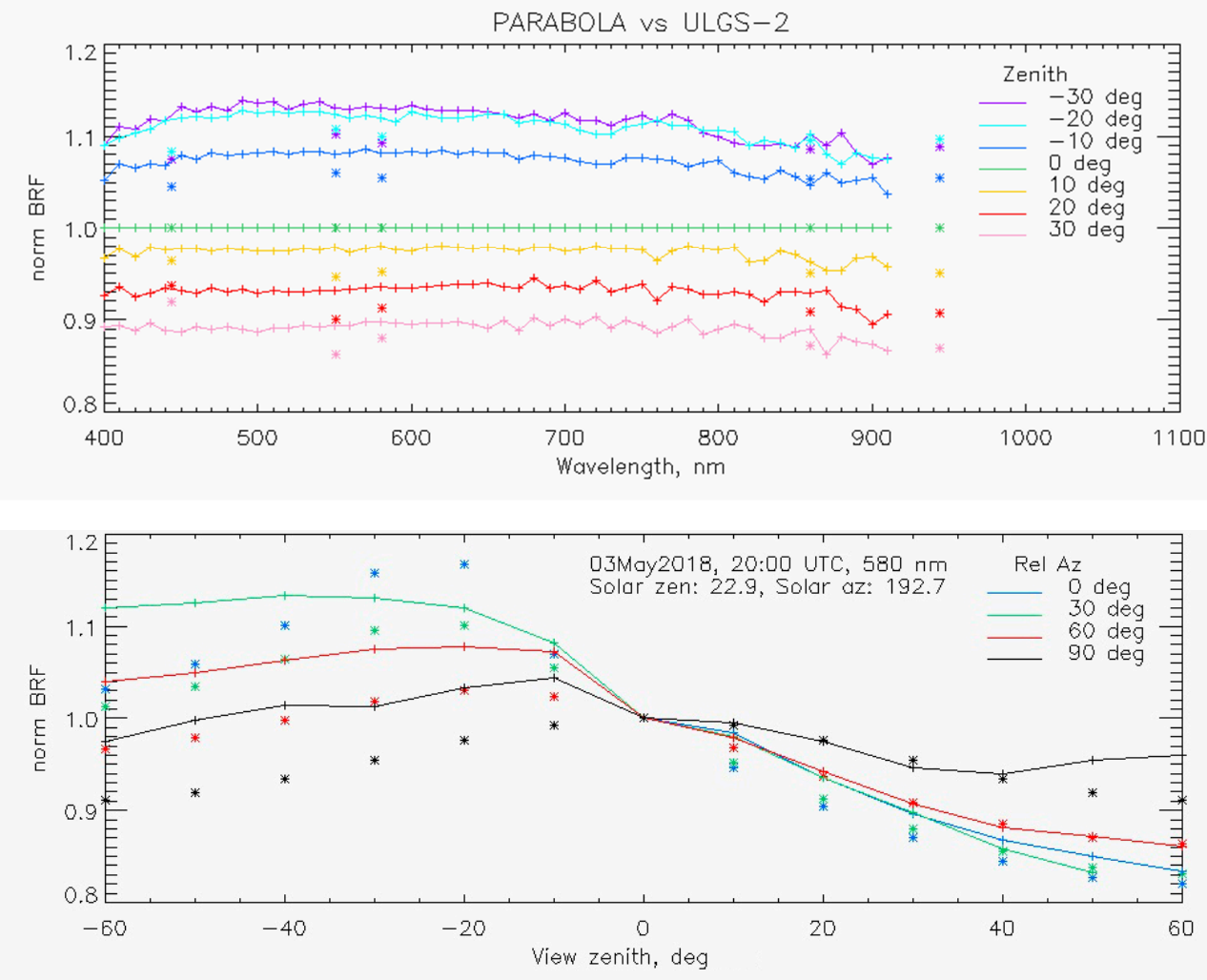

Figure 5. Comparison of normBRF versus wavelength for the PARABOLA (asterisk) and University of Lethbridge Goniometer System-2 (ULGS-2) (solid line) systems. (Top) normBRF versus wavelength at seven zenith views. (Bottom) normBRF versus view angle. The hotspot is in the principal plane, backscatter direction (negative zenith angles).

\section{Analytical Spectral Devices (ASD)}

\subsection{Transect Measurements}

The field spectrometer most often used to make nadir surface-reflectance measurements is a field portable spectroradiometer, dubbed ASD (after the manufacturer, Analytical Spectral Devices, now Malvern Panalytical (Longmont, CO, USA), model FieldSpecPro. This instrument is carried in a backpack and has a fiber optic cable that can be attached to a pole to extend the light-collecting end away from the field team collecting the data. The fiber is inserted into a pistol-grip, which also secures a small lens (the foreoptics) to the fiber. An $8^{\circ}$ field-of-view lens is used by the OCO team. Data, collected in linear traverses across an area as large as $500 \times 500 \mathrm{~m}$, are used to compute a spectral average. As the BRF varies with solar incident angle, it is important to collect data within a half hour of the satellite overpass. In addition to these nadir data collects, the ASD can be used in a number of ways to support off-nadir studies. The ULGS team makes use of an ASD, viewing nadir, to normalize the Ocean Optics USB4000 data. The ASD is used annually during the summer OCO-GOSAT VicCal 
campaign as an additional means to determine the off-nadir factor. This has been done using two techniques, as shown in Figure 6. In the first, the team walks a path, keeping the lens pointed at a number of discrete view angles, such as the $30 \mathrm{~W}$ or $20 \mathrm{E}$, which are the GOSAT Paths 37 and 36 , respectively. A digital inclinometer or simple protractor strapped to the pole holding the foreoptics can be used to maintain the desired angle. In the second approach, a target is placed on the ground, with marks indicating azimuth angle. This reference target, along with the protractor, is used to set the view azimuth and zenith angle of the ASD.
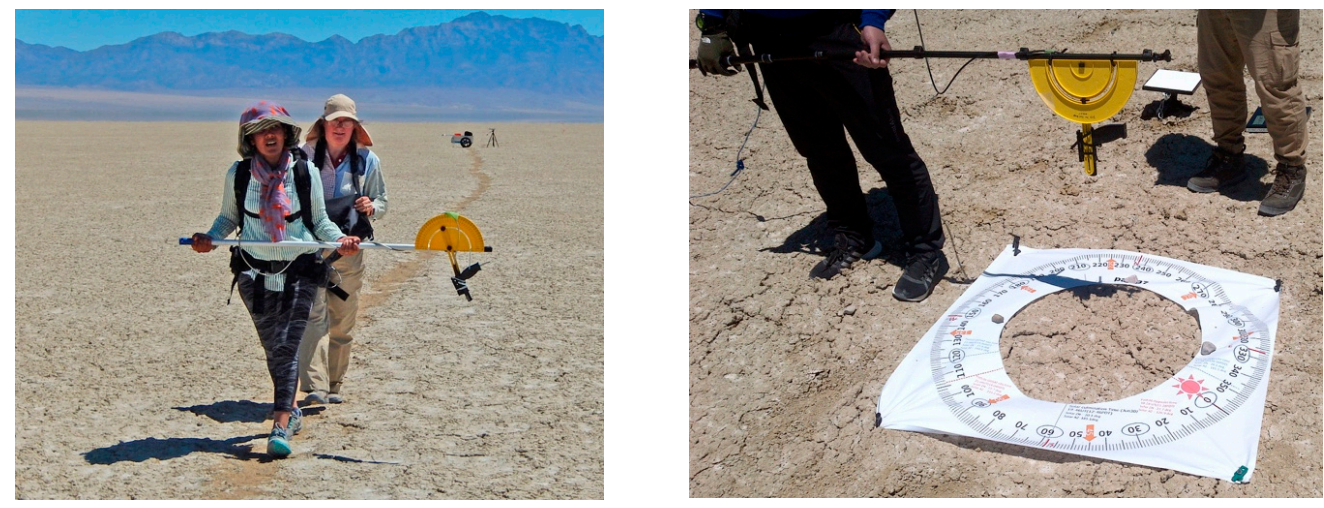

Figure 6. (Left) Protractor used to acquire off-nadir surface-reflectance data at Greenhouse gasses Observing SATellite (GOSAT) view angles; (Right) ground target used to identify view angles for off-nadir data collects.

Figure 7 shows the results of the first approach, where the field team acquired data over a $100 \mathrm{~m}$ path at a number of view zenith angles. It is shown that the spectral variation of normBRF is small compared with the variation in view angle. Whereas the change with wavelength is within $0.1 \%$ for view angles smaller than $30^{\circ}$, the variability with view angles ranges from 0.75 to 1.1 , a $32 \%$ range. These results are consistent with those from the PARABOLA, as reported in Table 2. At $551 \mathrm{~nm}$ the fractional difference between the ASD and PARABOLA is $1.5 \%$ at $30 \mathrm{~W}$ and $-2.2 \%$ at $20 \mathrm{E}$.
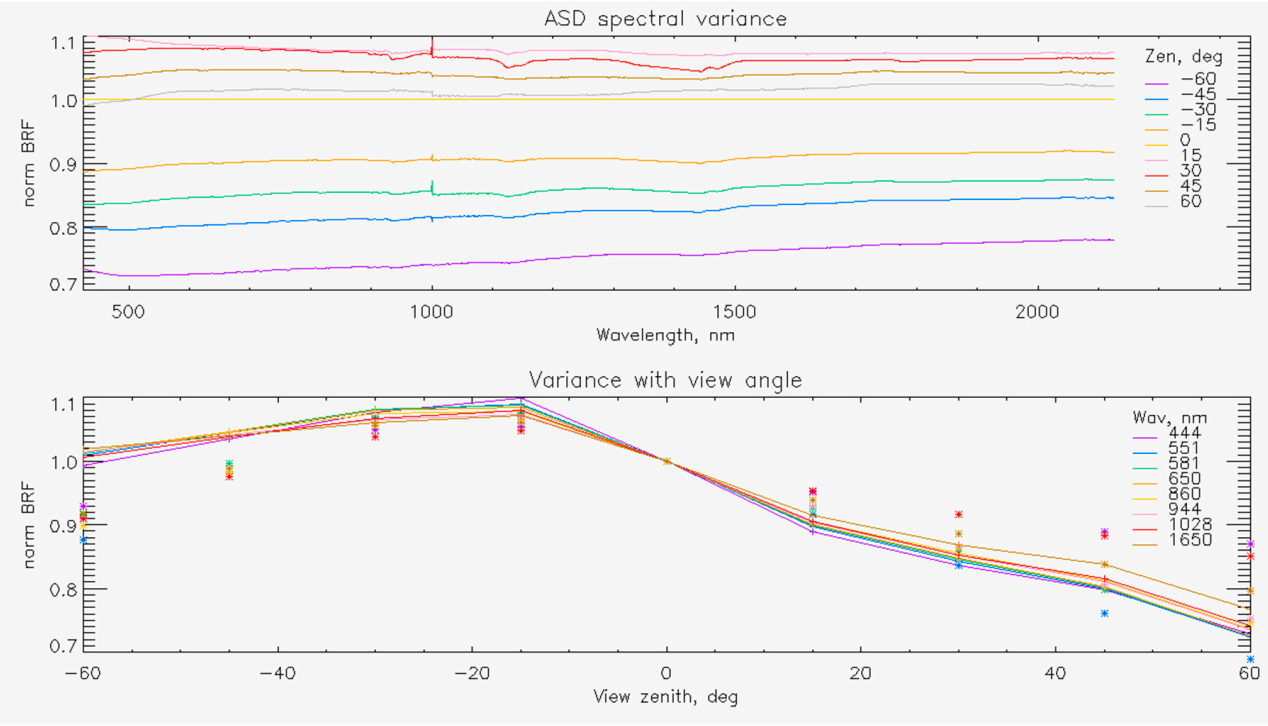

Figure 7. (Top) normBRF versus wavelength as determined by the ASD, with a number of view angles. Red lines are the backscatter, gold the nadir, and blues the forescatter directions. (Bottom) normBRF versus view zenith at PARABOLA wavelengths. PARABOLA (asterisk) and ASD data (solid lines) data are shown. 
Table 2. Comparison of PARABOLA normBRF versus ASD results at three PARABOLA wavelengths. The $444 \mathrm{~nm}$ PARABOLA channel suffers from low signal-to-noise, and the $650 \mathrm{~nm}$ channel was not operable for this campaign. This is for the MDN test site on 1 July 2018. Fractional difference is (normBRF_asd-normBRF_parab)/normBRF_parab.

\begin{tabular}{ccccccc}
\hline Instr & Band & $\mathbf{r 0}$ & $\mathbf{k}$ & $\mathbf{b}$ & $\mathbf{3 0 ~ W}$ & $\mathbf{2 0} \mathbf{E}$ \\
\hline PARAB & 444 & 0.198 & 0.917 & -0.012 & 1.050 & 0.952 \\
ASD & & & & & 1.076 & 0.889 \\
fracDiff & & & & 0.025 & -0.066 \\
\hline PARAB & 551 & 0.129 & 0.917 & -0.306 & 1.065 & 0.917 \\
ASD & & & & 1.081 & 0.897 \\
fracDiff & & & & & 0.015 & -0.022 \\
\hline ASD & 650 & & 0.918 & -0.218 & 1.080 & 0.900 \\
\hline PARAB & 860 & 0.209 & & & 1.059 & 0.929 \\
ASD & & & & & 0.015 & 0.906 \\
fracDiff & & & & & & -0.026 \\
\hline
\end{tabular}

\subsection{Goniometric Measurements}

A team of scientists from MAXAR Technologies (Westminster, CO 80234, USA) also participated in the RRV collaboration. On 4 May 2018, the MAXAR team collected ASD goniometric measurements within the RVUS RadCalNet Site just north of the University of Lethbridge Goniometer System. The setup is shown in Figure 8. The field spectrometer is fit with an $8^{\circ}$ field-of-view fore-optic lens and mounted on a tripod. The mount is kept at a consistent distance above the ground. An inclinometer is used to set the sensor view angle, while a marked hula-hoop is utilized to allow azimuthal alignment. Measurements are taken at $0^{\circ}, 5^{\circ}, 15^{\circ}, 20^{\circ}$, and $25^{\circ}$ view zenith at $30^{\circ}$ increments in azimuth. To avoid as much uncertainty from the atmosphere as possible, white references were taken often during measurement collection as the sky was filled with intermittent clouds on this day. Measurements were taken from 17:48:40-20:05:07 UTC (10:48:40-13:05:07 PDT). These data are then used to create a model of the surface reflectance at any sensor and sun angle set using the Rahman Pinty Verstraete (RPV) model, as was done with the PARABOLA data. Before creating the model, the data are examined, and any bad samples are removed. Bad samples may include a measurement accidentally including the hula-hoop, a person's shadow, or other unintended targets.
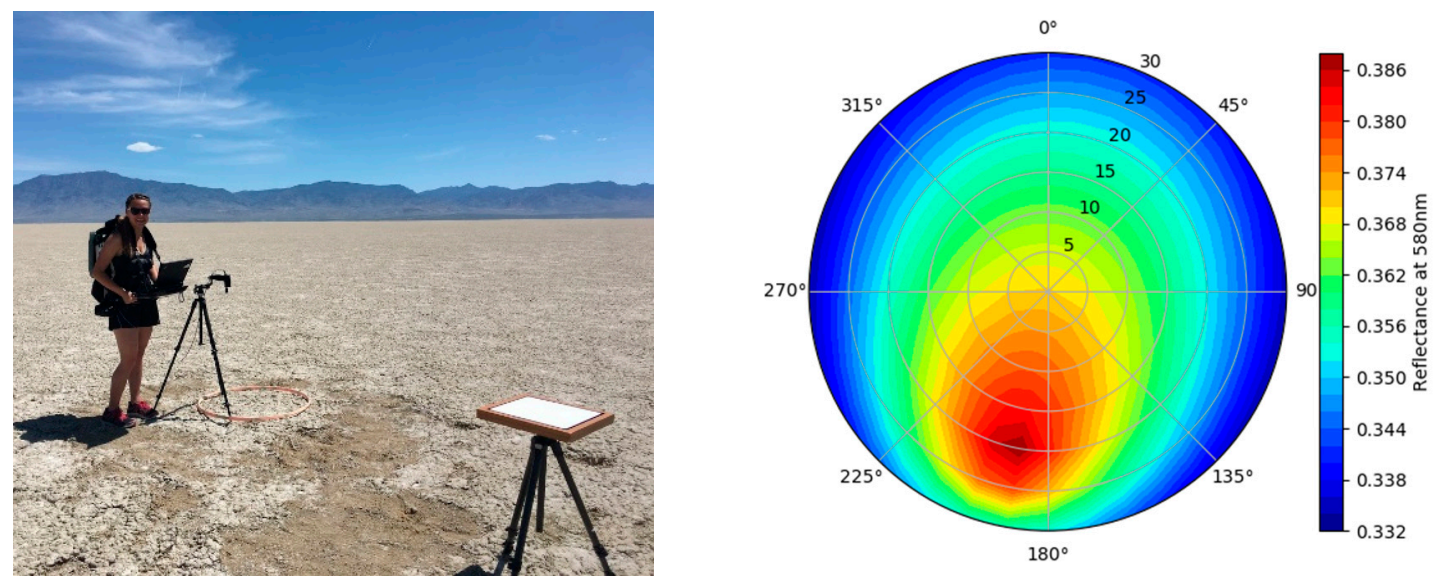

Figure 8. (Left) ASD goniometric measurements are taken on 4 May 2018. (Right) BRF at $580 \mathrm{~nm}$, 3 May 2018, 20:00 UTC. In addition, $13^{\circ}-193^{\circ}$ is the principal plane with the forward scatter (less reflectance) at the top and the hot spot at the backscatter towards the sun at $193^{\circ}$. The azimuth is given as $0^{\circ}$ North, $90^{\circ}$ East, $180^{\circ}$ South, $270^{\circ}$ West. The view angle is $0^{\circ}$ in the center and increases outwards. 
Azimuthal scans taken from the model data are shown in Figure 9, along with the PARABOLA data for the same view angles. Agreement is within 3\% for the forward scatter direction. The two sensors different in the hotspot direction, attributed perhaps to the ASD capturing fewer samples in this direction.

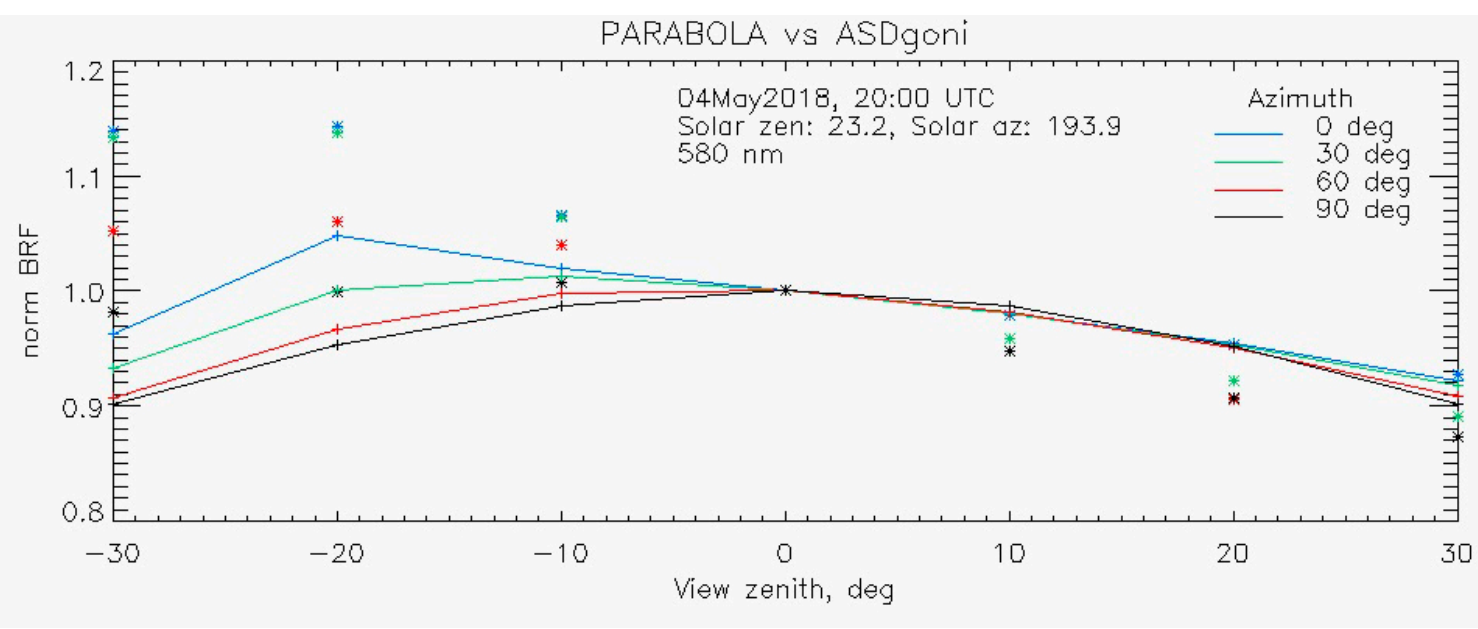

Figure 9. Plot of the Maxar empirical model output normBRF (solid line) versus view angle compared to PARABOLA (asterisk). The hotspot is in the principal plane, backscatter direction (negative zenith angles).

\section{MISR}

The MISR sensor, Figure 10, consists of nine cameras, viewing at nadir, $26.1^{\circ}, 45.6^{\circ}, 60.0^{\circ}$, and $70.5^{\circ}$ degrees forward and aftward of the local vertical. Each has spectral bands centered at 446, 557, 672, and $866 \mathrm{~nm}$. MISR data products include aerosol optical depth and composition, stereoscopically-derived cloud elevation, fraction, and texture, and surface BRF. To derive the latter, the top-of-atmosphere radiances are corrected for atmospheric effects, and the resulting surface reflectance fitted to retrieve coefficients of the mRPV surface model. This is the same functional form as used in the PARABOLA analysis. Results comparing MISR with PARABOLA are shown in Figure 11 and tabulated in Table 3. Ignoring the $444 \mathrm{~nm}$ PARABOLA band (with its low SNR), agreement is $1 \%$ or less at the two reference view angles.

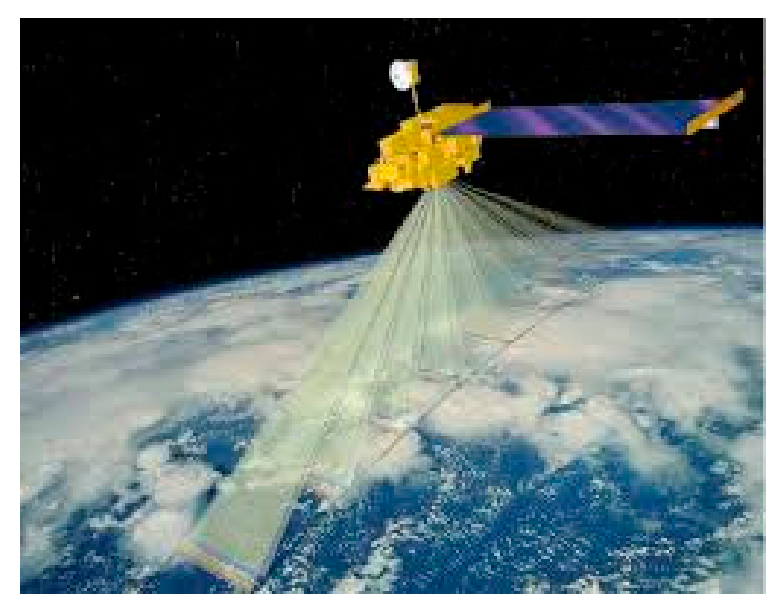

Figure 10. The Multi-angle Imaging SpectroRadiometer (MISR) instrument is one of five sensors on-board the Earth Observing System (EOS)/Terra spacecraft. Collecting data since early 2000, its radiometric response has been determined using VicCal campaigns. 


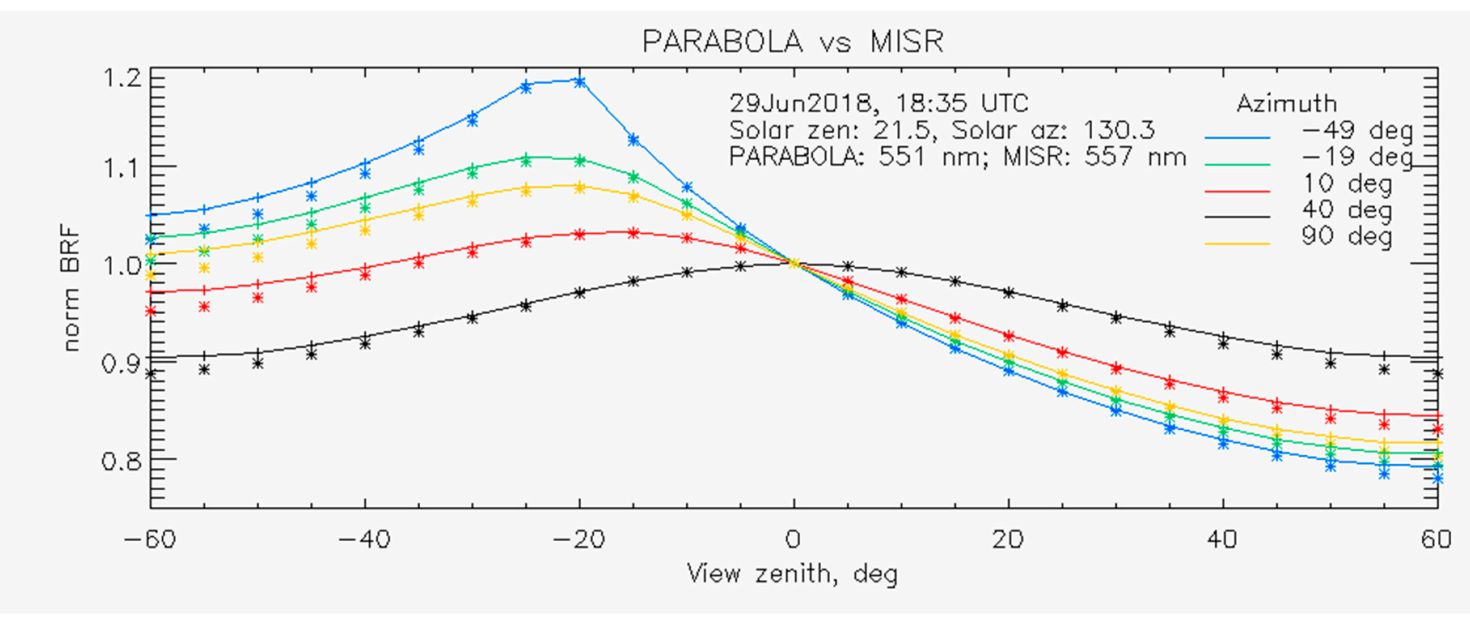

Figure 11. PARABOLA (asterisk) versus MISR (plus symbols and solid lines) normalized BRF at four azimuth angles. The view angles relative to the principal plane are $0^{\circ}, 30^{\circ}, 60^{\circ}, 90^{\circ}$, and $140^{\circ}$. Hotspot where normBRF $>1$ is in the backscatter direction, negative zenith angles.

Table 3. Comparison of PARABOLA normBRF versus MISR at M03 test site, 29 June 2018, GOSAT view angles. Fractional difference is (normBRF_misr-normBRF_parab)/normBRF_parab.

\begin{tabular}{ccccccc}
\hline Instr & Band & $\mathbf{r 0}$ & $\mathbf{k}$ & $\mathbf{b}$ & $\mathbf{3 0 ~} \mathbf{W}$ & $\mathbf{2 0} \mathbf{E}$ \\
\hline PARAB & 444 & 0.210 & 0.837 & -0.076 & 1.042 & 0.936 \\
MISR & 446 & 0.113 & 0.738 & -0.333 & 1.069 & 0.904 \\
fracDiff & & & & & 0.026 & -0.035 \\
\hline PARAB & 551 & 0.159 & 0.760 & -0.319 & 1.063 & 0.906 \\
MISR & 557 & 0.166 & 0.738 & -0.333 & 1.069 & 0.907 \\
fracDiff & & & & & 0.005 & 0.001 \\
\hline MISR & 672 & 0.094 & 0.667 & -0.595 & 1.069 & 0.905 \\
\hline PARAB & 860 & 0.231 & 0.782 & -0.230 & 1.057 & 0.921 \\
MISR & 866 & 0.207 & 0.738 & -0.333 & 1.069 & 0.909 \\
fracDiff & & & & & 0.011 & -0.013 \\
\hline
\end{tabular}

\section{MODIS}

The MODIS instrument images from both a morning and an afternoon platform, Terra, and Aqua, respectively. With its swath width of $2330 \mathrm{~km}(1448 \mathrm{mi})$, it can view a given location at a large range of view angles. A BRDF model is retrieved for each single day by combining observations from the two platforms over a 16-day period, which yields a sampling over the viewing hemisphere. The kernel-based RossThick-LiSparse Reciprocal model is used to produce the coefficients f_iso, f_vol, f_geo, which relate to isotropic, volumetric scattering, and geometric-optical scattering kernels, respectively. These are reported in the land surface BRDF product, MCD43A1. The associated data quality product, MCD43A2, indicates whether the full, high quality BRDF inversion was performed for a given pixel, or whether a lower quality magnitude inversion was performed instead, due to an insufficient number and inadequate angular sampling of observations. Note that a very similar BRDF product is generated using data from the Visible Infrared Imaging Radiometer Suite (VIIRS) sensor for both the imaging bands (VNP43IA1) and the moderate resolution bands (VNP43MA1). This product relies on the single VIIRS instrument currently available aboard the SNPP platform, which has afternoon overpasses similar to Aqua. BRDF retrievals for both MCD43A1 and VNP43M/IA1 are reported over a 16-day sliding window, with the heavily weighted day of interest being the 9th day in the window. The data quality flag has a range of $0-4$, with increasing uncertainty. A value 
of 0 indicates that a high-quality full retrieval was possible; 4 indicates a failed retrieval, with a fill value replacing the data product. Furthermore, extensive quality flags are also available on a per band basis. In attempting to use the standard MODIS product over RRV, it became clear that the retrieval algorithm had difficulties in using the surface reflectance values (MO/YD09) due to the bright playa surface. This resulted in a misclassification of the scene as cloudy or having an aerosol haze. Of the 32 overpass dates investigated, all associated with field campaigns where clear-sky conditions prevailed, the MCD43A1 product frequently failed to provide the BRDF coefficients. Results are shown in Figure 12. Specifically, H14 reported fill values (a non-retrieval) for all but one overpass date; sites M03 and M20 had valid data for only $20 \%$ of the dates.

As an alternative to the standard product, the University of Massachusetts Boston team has produced a special product, relaxing the aerosol flag from the input surface reflectance observations (MO/YD09), thereby avoiding the misclassification, and providing valid data for all four sites. An example of this retrieval, as compared to PARABOLA, is shown in Figure 13 and Table 4.

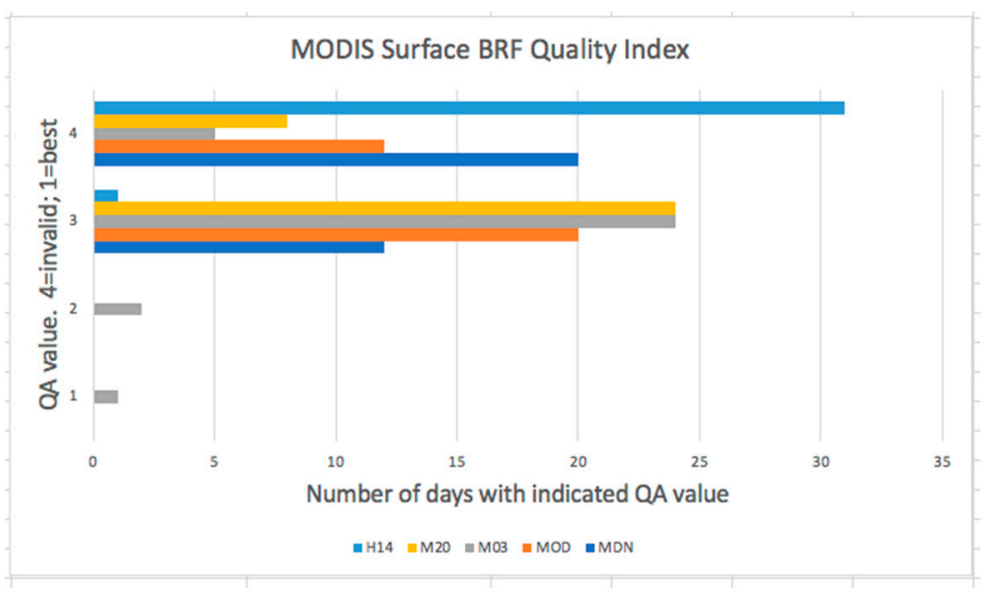

Figure 12. Quality Assurance (QA) flag as reported over 32 Railroad Valley collection dates and four locations, all within the playa.

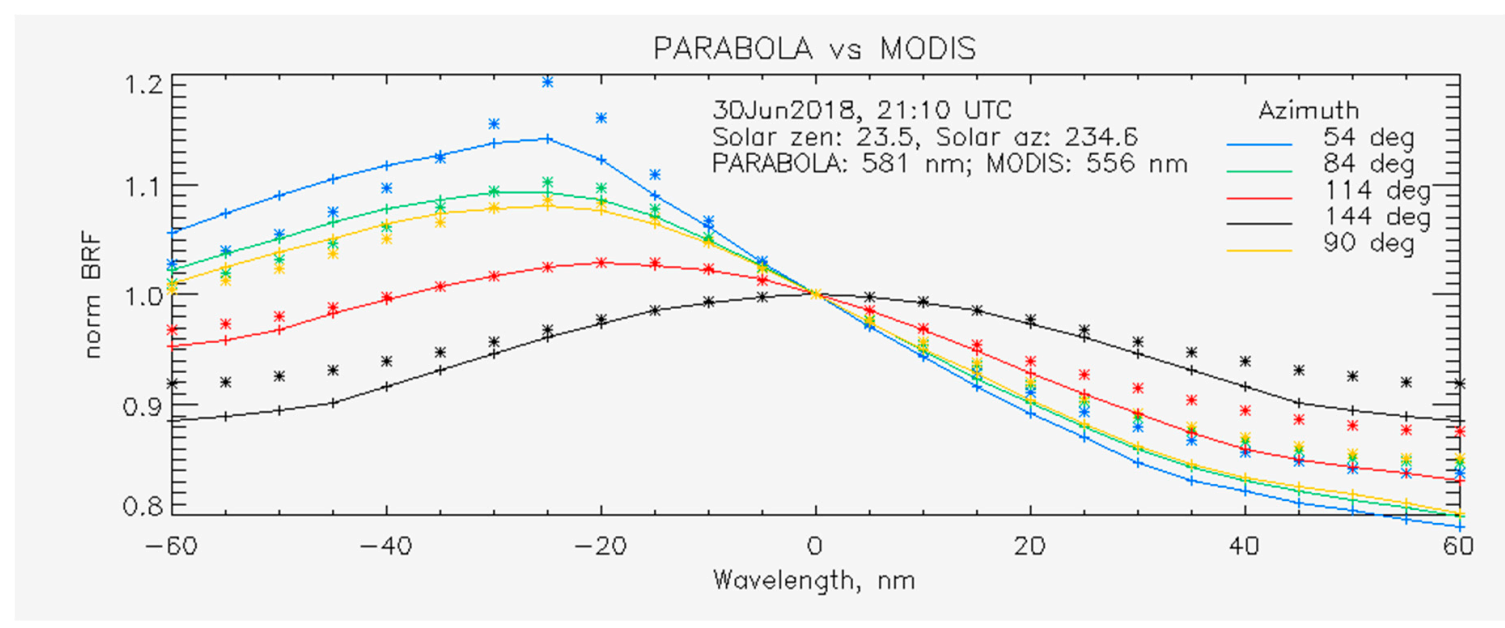

Figure 13. Normalized BRF as measured by PARABOLA and MODerate resolution Imaging Spectroradiometer (MODIS) on 30 June 2018, site M03. Curves represent a number of view azimuths relative to the Sun. The largest deviation from unity is shown to be in the backscatter direction of the principal plane. 
Table 4. Comparison of PARABOLA normBRF versus MODIS at Greenhouse gasses Observing SATellite (GOSAT) view angles. Comparison is for site M03, with PARABOLA data acquired on 29 July 2018 and MODIS 30 June 2018. Fractional difference is (normBRF_modis-normBRF_parab)/normBRF_parab.

\begin{tabular}{cccc}
\hline PARAB Band, $\mathbf{n m}$ & MODIS Band, $\mathbf{n m}$ & FracDiff $\mathbf{3 0} \mathbf{~}$ & FracDiff 20 E \\
\hline 444 & 446 & 0.023 & -0.053 \\
551 & 556 & -0.002 & -0.009 \\
860 & 862 & 0.016 & -0.037 \\
1650 & 1631 & 0.011 & -0.029 \\
\hline
\end{tabular}

\section{Conclusions}

Vicarious calibration using Railroad Valley has an uncertainty of 3\% for nadir-viewing sensors, under ideal clear-sky conditions. The uncertainty grows for sensors that view the test site at off-nadir view angles. If a nadir-measured surface reflectance is used in the calibration, without correction for BRF with view angle, an error of up to $10 \%$ can be incurred for sensors that view up to $30^{\circ}$ off-nadir. Data from eight years of field measurements using the PARABOLA instrument show that the BRF, normalized to its nadir value (normBRF), is stable across the playa and from one year to the next to with a few percent at the GOSAT view angles of $30^{\circ}$ from the West and $20^{\circ}$ from the East. The off-nadir correction term can also be obtained from the MISR Level 2 Surface product, where the normBRF agree with PARABOLA to within 1\%. The excellent agreement between these two sensors may be partially attributed to the fact that they use the same empirical model to fit the measurements. MISR BRF data may be of interest to those using other desert sites, such as the Saharan desert, for cross-sensor comparisons. The MODIS surface BRF product is most likely to fail over the bright RRV playa. However, a special MODIS product, which is produced ignoring the aerosol QA flag, is found to agree with PARABOLA to within $4 \%$. In comparing PARABOLA data to the ULGS-2 instrument, we note neither measure the hotspot directly, but both instruments showed similar results. This similarity is significant as the instruments are measuring in opposite modes: one at a fixed location scanning the surrounding area and the other moving on a goniometer frame looking at a fixed location. This provides further confirmation of the consistency of the RRV BRF. As PARABOLA analysis fits the data to a model that retrieves the hotspot, the missing data are provided. We postulate that the PARABOLA $r_{0}, b$, and $k$ coefficients reported here can be used to compute normBRF for an arbitrary sun and view geometry at RRV, and that these are good to within a few percent, provided the surface conditions are dry (no rain within the past week, and no snow melt). That is, the "typical" coefficient set is representative of the playa under dry-soil conditions, irrespective of location on the playa, but is likely to be invalid following rain or snow. Making use of the off-nadir correction term, the VicCal can be obtained for OCO and GOSAT sensors to within their uncertainty budget of $5 \%$.

Author Contributions: PARABOLA data analysis and cross-instrument comparisons were performed by C.J.B.; C.C. designed, built, and processed data from the ULGS; C.S. and A.E. provide special MODIS BRF data products, with relaxed cloud screening; M.C.H. assisted in the initial build of the PARABOLA, and has maintained this instrument over a number of decades; F.K. has studied BRF from MODIS and VIIRS, in support of GOSAT calibration; A.K. and K.S. have co-assisted in carrying out the annual OCO-GOSAT field campaigns; F.M.S. has been responsible for PARABOLA operation, including mast installation, and has also contributed as mineralogy expert for surface materials on the Railroad Valley playa; M.K. and T.O. provided ASD goniometric measurements.

Funding: GOSAT and GOSAT-2 are joint projects of the Japan Aerospace Exploration Agency (JAXA), the National Institute for Environmental Studies, and the Ministry of the Environment (MOE) of Japan, collectively called the "GOSAT Partners". They participate in RRV campaigns under funds provided by their respective employers. Funding for the OCO-2 team participation described in this paper was carried by staff from the Jet Propulsion Laboratory, California Institute of Technology, under a contract with the National Aeronautics and Space Administration. MAXAR scientists participate in RRV campaigns under funds provided by their employer. RRV is under Bureau of Land Management (BLM) stewardship, a Federal agency whose mission is to coordinate shared usage of public land. Both NASA/JPL and the University of Arizona, Tucson, have land rights-of-way (ROW) with the BLM, in order to place network sensors on the playa. 
Acknowledgments: The OCO and GOSAT team have participated in an annual joint VicCal campaign since 2009. The GOSAT team lead has been Akihiko Kuze, with support from Kei Shiomi. Many summer interns have assisted in data collection, including Suniti Sanghavi (2017 campaign), pictured in Figure 6, and the then Caltech undergraduate student Sharon Yang (2013 campaign, now at Google LLC, City, US State abbrev., Country), who contributed to the PARABOLA data analysis code. David Crisp, Robert A. Rosenberg, and Annmarie Eldering have provided support and management for the OCO effort. Linley Kroll provides invaluable support during the campaigns, including setup, instrument repair and trouble-shooting, and power. Michael Bull has provided MISR surface reflectance coefficients. Kurt Thome and Jeff Czapla-Myers have organized the May 2018 campaign which brought together a number of VicCal scientists.

Conflicts of Interest: The authors declare no conflict of interest.

\section{References}

1. Bruegge, C.; Chrien, N.; Ando, R.; Diner, D.; Abdou, W.; Helmlinger, M.; Pilorz, S.; Thome, K. Early validation of the Multi-angle Imaging SpectroRadiometer (MISR) radiometric scale. IEEE Trans. Geosci. Remote. Sens. 2002, 40, 1477-1492. [CrossRef]

2. Bruegge, C.J.; Crisp, D.; Helmlinger, M.C.; Kataoka, F.; Kuze, A.; Lee, R.A.; McDuffie, J.L.; Rosenberg, R.A.; Schwandner, F.M.; Shiomi, K.; et al. Vicarious Calibration of Orbiting Carbon Observatory-2. IEEE Trans. Geosci. Remote. Sens. 2019, 57,5135-5145. [CrossRef]

3. Briere, P.R. Playa, playa lake, sabkha: Proposed definitions for old terms. J. Arid. Environ. 2000, 45, 1-7. [CrossRef]

4. Bouvet, M.; Thome, K.; Berthelot, B.; Bialek, A.; Czapla-Myers, J.; Fox, N.P.; Goryl, P.; Henry, P.; Ma, L.; Marcq, S.; et al. RadCalNet: A Radiometric Calibration Network for Earth Observing Imagers Operating in the Visible to Shortwave Infrared Spectral Range. Remote. Sens. 2019, 11, 2401. [CrossRef]

5. RadCalNet Portal. Available online: https://www.radcalnet.org/\#!/ (accessed on 27 October 2019).

6. Bruegge, C.J.; Val, S.; Diner, D.J.; Jovanovic, V.; Gray, E.; Di Girolamo, L.; Zhao, G. Radiometric stability of the Multi-angle Imaging SpectroRadiometer (MISR) following 15 years on-orbit. Earth Observing Systems XIX 2014, 9218, 92180N.

7. Kuze, A.; O’Brien, D.M.; Taylor, T.E.; Day, J.O.; O’Dell, C.W.; Kataoka, F.; Yoshida, M.; Mitomi, Y.; Bruegge, C.J.; Pollock, H.; et al. Vicarious Calibration of the GOSAT Sensors Using the Railroad Valley Desert Playa. IEEE Trans Geosci Remote Sens. 2011, 49, 1781-1795. [CrossRef]

8. Kuze, A.; Taylor, T.E.; Kataoka, F.; Bruegge, C.J.; Crisp, D.; Harada, M.; Helmlinger, M.C.; Inoue, M.; Kawakami, S.; Kikuchi, N.; et al. Long-Term Vicarious Calibration of GOSAT Short-Wave Sensors: Techniques for Error Reduction and New Estimates of Radiometric Degradation Factors. IEEE Trans Geosci Remote Sens. 2014, 52, 3991-4004. [CrossRef]

9. Bruegge, C.J.; Helmlinger, M.C.; Conel, J.E.; Gaitley, B.J.; Abdou, W.A. PARABOLA III: A sphere-scanning radiometer for field determination of surface anisotropic reflectance functions. Remote. Sens. Rev. 2000, 19, 75-94. [CrossRef]

10. Martonchik, J.V.; Bruegge, C.J.; Strahler, A.H. A review of reflectance nomenclature used in remote sensing. Remote. Sens. Rev. 2000, 19, 9-20. [CrossRef]

11. Diner, D.J.; Martonchik, J.V.; Borel, C.; Gerstl, S.A.W.; Gordon, H.R.; Knyazikhin, Y.; Myneni, R.; Bernard, P.; Verstraete, M.M. Level 2 Surface Retrieval Algorithm Theoretical Basis. Available online: https://eospso.gsfc. nasa.gov/atbd-category/45 (accessed on 08 May 2019).

12. Rahman, H.; Verstraete, M.M.; Pinty, B. Coupled surface-atmosphere reflectance (CSAR) model: 1 . Model description and inversion on synthetic data. J. Geophys. Res. Space Phys. 1993, 98, 20779. [CrossRef]

(C) 2019 by the authors. Licensee MDPI, Basel, Switzerland. This article is an open access article distributed under the terms and conditions of the Creative Commons Attribution (CC BY) license (http://creativecommons.org/licenses/by/4.0/). 